\title{
(C) OPEN ACCESS \\ Cultural understanding in the provision of supportive and palliative care: perspectives in relation to an indigenous population
}

\author{
Grace Johnston, ${ }^{1}$ Adele Vukic, ${ }^{2}$ Skylan Parker ${ }^{3}$
}

\begin{abstract}
${ }^{1}$ School of Health Administration, Dalhousie University, Halifax, Canada ${ }^{2}$ School of Nursing, Dalhousie University, Halifax, Canada ${ }^{3}$ Department of Health and Social Services, Government of Yukon, Whitehorse, Canada
\end{abstract}

\section{Correspondence to}

Grace Johnston, School of Health Administration, Dalhousie University, 5161 George Street, Suite 700 - Royal Centre PO Box 15000, Halifax, Nova Scotia B3H 4R2, Canada; Grace.Johnston@dal.ca

Accepted 24 February 2012 Published Online First 3 May 2012

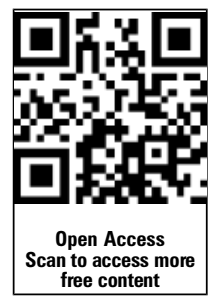

To cite: Johnston $G$, Vukic $A$, Parker S. BMJ Supportive \& Palliative Care 2013;3:61-68.

\section{ABSTRACT}

Objectives The provision of supportive and palliative care for an indigenous people in Nova Scotia, Canada, was examined to further our understanding and thereby improve cultural competency. Most of Nova Scotia's indigenous people are Mi'kmaq. The Mi'kmaq Nation lives in Atlantic Canada as well as New England in the eastern USA.

Methods Themes were identified in the literature and through discussion with seven experts who have Mi'kmaq health and cultural research expertise. This paper has been reviewed and approved by two Mi'kmaq consultants who frequently speak on behalf of the Mi'kmaq people in relation to health and cultural understanding. Recommendations for nonindigenous care providers are presented.

Results The themes identified focused on jurisdictional issues and cultural understanding. They are interconnected and grounded in the historic Mi'kmaq context of colonialism. Jurisdictional issues experienced by the Mi'kmaq affect access, continuity and appropriateness of care. Cultural concepts were associated with worldview, spirituality, the role of family and community relationships and communication norms, and thereby with the alignment of values and language in the provision of care. Three Mi'kmaq concepts are noted: apiksiktatulti, nemu'ltus and salite.

Conclusion Through reflection on the situation of Nova Scotia's Mi'kmaq, non-indigenous healthcare providers can assess how they might increase their cultural understanding in the provision of supportive and palliative care. Recommendations relate to the health system, relationships with individual persons and direction for research.

Conceptualisations of death and dying, and emotional expressions of grief and loss, are largely socially constructed. Therefore, the ability to adequately understand cultural values and practices is essential when providing supportive and palliative care. ${ }^{1}$ Recognition of an individual's needs, values and beliefs within a cultural context supports the autonomy and human rights of the persons receiving care. ${ }^{2}$ Cultural differences in attitudes toward providing comfort, truth-telling, decision-making including family roles, understanding of the meaning of the end of this physical life, and using indigenous practices and rituals reinforce the need for cultural understanding. ${ }^{2}$ Cultural understanding leads to cultural competence as healthcare professionals begin to recognise the interests, emotions and biases that are a part of their own culture, ${ }^{3}$ since culture is only meaningful when interpreted in the context of an individual's belief and value system. ${ }^{4}$ The more opportunity that care providers have to build meaningful relationships with persons approaching the end of this life, particularly with persons whose culture differs from one's own, the greater the likelihood that persons will receive comprehensive and compassionate care.

In this paper, Mi'kmaq understandings and recommendations are presented for consideration to enhance culturally competent supportive and palliative care. In Canada, indigenous peoples include the Inuit, Metis and status and non-status First Nations. They are collectively known as the Aboriginal peoples. In 2006, there were an estimated 24175 Aboriginal persons living in the province of Nova Scotia, which is $2.6 \%$ of the population of the province. ${ }^{5}$ The 
Mi'kmaq Nation lives in Atlantic Canada as well as New England in the eastern USA. The majority of Nova Scotia's Aboriginal population belong to the Mi'kmaq Nation and reside in 13 First Nations communities. Mi'kmaq is the collective noun of the singular form and adjective Mi'kmaw.

Mi'kmaq perspectives of support and healthcare as they relate to the provision of supportive and palliative care are identified and discussed both from a historical point of view as well as in the current context of care. Tribal differences withstanding, indigenous peoples report that indigenous knowledge is often not understood or is considered only through the lens of Western healthcare practice. ${ }^{6}$ Furthermore, there is now a recognition that non-indigenous society can learn much from indigenous community-based practice at the end of life. ${ }^{7}$

This paper provides a synthesis of traditional views of life and death of the Mi'kmaq in the context of the inter-relationship between the collective community and personal beliefs and values. Although the context is that of the Mi'kmaq, cultural understanding should be considered with all indigenous cultures and for non-indigenous peoples. This manuscript provides non-indigenous healthcare providers and researchers with a situation for reflection on cultural understanding in the provision of care.

\section{METHODS}

This paper was developed from a review of literature and discussions of one author (SP) with four non-Mi'kmaw informants intricately involved with research and Mi'kmaq peoples and the second author (AV) who shared this paper with two Mi'kmaw consultants (Theresa Meuse, advisor and educator on Mi'kmaq ways, and Sister Veronica Matthews, Elder). This study was carried out in the context of respectful Aboriginal ways which value relationships and begins by attaining an understanding of the context and history. ${ }^{8}$

The authors are not from the Mi'kmaq Nation. GJ leads research to improve access to quality care for populations at end of life. AV practiced as a nurse with the Aboriginal people and has taught northern nurse practitioners. SP was a graduate student with GJ and AV. SP worked under the direction of AV in meeting one-to-one with each of the non-Mi'kmaw informants whose research is recognised and valued by the Mi'kmaw people but who do not have the authority to speak on behalf of the Mi'kmaw people. Therefore, ensuring the authority to speak of Mi'kmaw ways was essential.

AV has worked with two Mi'kmaw consultants (TM, SVM) for more than 20 years. TM and SVM are recognised spokespersons for the Mi'kmaq community particularly in relation to health issues. TM and SVM reviewed the paper prior to submission for publication, and they reviewed and approved revisions in response to journal reviewer comments.

Table 1 provides a description of key Mi'kmaq terms, as well as cultural competency, and supportive and palliative care as used in Nova Scotia. Throughout the process of developing this paper, there was an ongoing need to specify and clarify the meaning of terms.

\section{RESULTS}

The themes that emerged are interconnected and grounded in the historic Mi'kmaq context of colonialism. The role of colonialism is included in table 2 but not developed further because it has been well documented by Twohig. ${ }^{9}$ The second theme is jurisdictional issues which relate to the three systems of support for the Mi'kmaq: federal, provincial and traditional indigenous. Third, cultural understanding is needed in relation to both values and language.

\section{Jurisdictional issues in providing palliative and supportive care for the Mi'kmaq}

The Canadian federal government has responsibility for the Aboriginal peoples. However, the delivery of health services in Canada is primarily a provincial responsibility. There are gaps and differences in services that are funded by each jurisdiction.

Supportive ${ }^{10} 11$ and palliative care have not been core services of Indian and Northern Affairs Canada (INAC), the department which has the responsibility of meeting the Government of Canada's obligations for First Nations peoples through negotiations with them. Most Mi'kmaq communities have community health nurse(s) who are involved with families in providing care. Health Canada and INAC together developed a National Framework on Home Care for First Nations and Inuit communities using this definition: 'a system of service delivery encompassing a range of insured, extended and uninsured health and social services for all age groups, addressing the wholistic, social and personal care needs of individuals who do not have, or have lost, some capacity for self-care. ${ }^{12}$ The INAC adult care programme can meet some supportive and palliative care needs. INAC may pay to provide care services including laundry, meals and housekeeping. To respect client preferences, family members may provide these services. Personal care can include pain management, spiritual support and comfort measures, familial support and care planning related to disease as well as the settling of personal affairs. Personal care workers funded by INAC have some training in personal care.

The lack of extensive educational and employment opportunities for Aboriginal care givers reinforces marginalisation and results in female Aboriginal caregivers being disadvantaged socially and politically. ${ }^{13}$ Other issues include coping with the stress of physical illness, the role of the family in treatment and 
Table 1 Descriptions of terms related to providing Mi'kmaq supportive and palliative care

\begin{tabular}{l} 
Term \\
\hline Aboriginal peoples \\
Mi'kmaq \\
Apiksiktatultimk (pronounced \\
abiksikdadldimk)
\end{tabular}
abiksikdadldimk)

Nemu'Itus

Salite

Cultural competency

Palliative care

Supportive care

Indian and Northern affairs

\section{Description}

Indigenous people who have lived in Canada for thousands of years.

First Nation people whose name roughly translates as 'the family'. Oral tradition is important in the relational nature of the Mi'kmaq language.

A Mi'kmaq term used to describe when a person is thought to be dying, family and friends go to the bedside to partake in this act of mutually being present with each other which may include forgiveness or reconciliation. This has the intent of ensuring that the dying person will go to the spirit world without any burden while also preparing all involved for the inevitable.

A commonly used Mi'kmaq saying which translates to 'I'll see you.' It is mostly used as a form of goodbye, but is also used when someone is dying. The implication here is that death is not final. Life and death are events. Death is understood to be a verb since if it were a noun, it would be final. Life is also a verb as it is a process of living and of being alive.

Mi'kmaq feast after a person leaves this world. Feasts are common for First Nations peoples for closure after events. Usually the Elders are the first to begin the meal. This is a community event with everyone bringing goods for an auction. In the past, the Salite auctioned the belongings of the person who has gone to the spirit world. Today, community members bring goods to auction to help raise funds to pay for the wake and other expenses and debts the person may have had. Salite is an important aspect of care. It acknowledges the interconnectedness of the person who has moved on to the spirit world and the community.

As a continuum, cultural competency encompasses sensitivity, awareness, knowledge, skills and competency. Cultural competency depends on provider efforts to understand unique aspects of individual cultures and is based on the premise that knowledge of cultural characteristics equips professionals to build trusting relationships and provide holistic care.

Palliative care aims to improve the quality of life and care of patients and their families facing life-threatening illness through the prevention and relief of suffering by early intervention and treatment of pain and other physical, psychosocial and spiritual problems. In Canada, palliative care and palliative medicine are typically hospital-based and provincially organised. Palliative care developed in Canada during a time period when federal-provincial agreements provided public funding for hospital and physician care, and patchwork funding at best for community-based care. The role of the voluntary sector and the federal government is minimal in the delivery of palliative care in Canada.

Supportive care relates primarily to cancer services that help patients and families with their experience during the diagnostic, treatment, follow-up and palliative phases. It includes physical and symptom support as well as information, psychological and spiritual support.

INAC is the Canadian federal government department with a mandate to fulfil constitutional responsibilities in First Nations affairs regarding the Indian Act which was established in 1867. Since 1949, matters of healthcare and First Nations have been directed to the federal department of Health Canada. INAC's name was changed to AANDC in 23 June 2011. AANDC is responsible for economic development and social well-being.

AANDC, Aboriginal Affairs and Northern Development Canada; INAC, Indian and Northern Affairs Canada.

decision-making, financial burdens, transportation to health services and provision of support for emotional and spiritual health. A lack of quality housing is also recognised as a fundamental challenge in providing adequate home-based care. ${ }^{12}$

The Mi'kmaq population is primarily rural and small. Therefore, choices are limited. Beyond INAC and Health Canada services, some communities have a relationship with a provincially funded palliative care programme to assist in managing pain and other symptoms. Each of the nine district health authorities across Nova Scotia operates a palliative care programme. Their evolution is comparable with that in other Canadian provinces including greater gaps in

Table 2 Themes emerging from a review of literature and discussion with informants on culturally competent supportive and palliative care for the Mi'kmaq

\begin{tabular}{ll}
\hline Theme & Description \\
\hline Historic context of & It is critically important to recognise the role of colonialism in shaping policy and its impact on what services are available to \\
colonisation & Canada's First Nation peoples. Unlike other parts of the country, there has been no land surrender in Nova Scotia. \\
Jurisdictional issues & $\begin{array}{l}\text { Continuity of care may be lost as individuals require care outside their community and move between provincial and federal } \\
\text { services. } \\
\text { Cultural understanding }\end{array}$ \\
$\begin{array}{l}\text { Values: lack of alignment between values implicit in Western medicine and those of the Mi'kmaq culture can be problematic. } \\
\text { Using a holistic health approach can act as a bridge for understanding indigenous values such as the interconnectedness of } \\
\text { the physical, social and spiritual dimensions of self and the impact this has on health decisions. Rather than being universal } \\
\text { and unchanging, values differ, often subtly, by family and individual as well as from community to community. } \\
\text { Language: acknowledging culture as a part of an individual's healing process is vital to understanding health choices and } \\
\text { preferences. The integration of spirit as a part of healing occurs through the inseparable interrelationships among self, family } \\
\text { and community. Different world views, language and cultural contexts reflect the divide that needs to be bridged to attain } \\
\text { cultural competency in the care of indigenous peoples at end of life. Complexity is inherent in gaining a deep understanding } \\
\text { of language. }\end{array}$
\end{tabular}


rural than in urban areas. ${ }^{14}$ Their extent of services varies as does their linkage to supportive cancer care $^{15}$ and to the provincial home care programme. While the Nova Scotia Department of Health and Wellness recognises the importance of cultural competency in the provision of healthcare, ${ }^{16}$ concerns remain.

Jurisdictional issues are compounded by Treaty and non-Treaty status and First Nations people living on and off reserve. Issues include transportation to and from treatment areas and associated costs and financial restrictions for accessing certain services. Costs may depend on the length of time individuals are away from home accessing services. Feelings of loneliness and isolation result from unfamiliar environments and contribute to social exclusion. ${ }^{17}$

A government report ${ }^{16}$ states that provincial hospital services are poorly responsive to Aboriginal healing practices. Difficulty in recruiting and retaining on-reserve physicians and poor relationships with offreserve provincial health professionals are issues that the Mi'kmaq representatives identified. Even though it may appear that increased funding is being committed to improving Aboriginal health, the proportion per capita is still much less than what is spent per person in the rest of Canada. ${ }^{18}$ Disparities in access, quality care and health outcomes as well as provider biases, lack of continuity of care, healthcare system barriers and limited interpretation services are similar to reports for indigenous peoples elsewhere in North America. ${ }^{12}$ Jurisdictional issues experienced by the Mi'kmaq affect access, continuity and appropriateness of care. Many of these issues are connected with the impact of the historic residential school system which did not value Mi'kmaq ways, created intergenerational trauma, and led to disruption of culture, language, traditions and history. This parallels a bias that has been reported for some primary healthcare providers who appear disrespectful and have a poor knowledge or understanding of First Nations culture and misconceptions around 'compliance'. ${ }^{16}$ Great imbalances of power exist in terms of the degree of decision-making that is enabled within the Aboriginal communities. Table 3 provides recommendations for practice at the level of the healthcare system and the individual requiring care.

\section{Cultural understanding in the provision of supportive and palliative care \\ Values}

The lack of alignment between the values implicit in Western medicine and indigenous peoples is problematic. Cultural understanding can act as a bridge to accepting Aboriginal values such as the interconnectedness of the physical, social and spiritual dimensions of self and the impact this has on health decisions. Acknowledging culture as a part of an individual's healing process is vital to comprehending health

\section{Table 3 Recommendations for non-indigenous care providing supportive and palliative care}

\section{The systems: learn history of indigenous people and relevance to healthcare today}

(A) Be aware and adapt to the impact of the historic Even though practices may differ today, pain from the past reverberates and retains a resonance context of colonialism.

(B) Recognise the gaps and issues in the provision of indigenous health services.

\section{The individual: gain insight into the views of the person}

that must be recognised. Colonialism shaped political relationships and resources and led to discrimination and injustices including lack of respect and unequal access to care.

While not a main focus of this paper, addressing health system problems is essential for the provision of culturally competent supportive and palliative care. The need to lobby to improve access, quality, comprehensiveness and continuity of care is apparent.

(A) Make an effort to get to know the individual.

Cultural needs must be considered on an individual basis, not according to cultural assumptions or biases. Individuals are unique, even within their cultures. Dignity is individually determined. Providers should take every opportunity to build a relationship with the patient/client to enable them provide competent care. Cultural knowledge and awareness is one step in the continuum of providing quality care.

(B) Treat each situation as unique and case-specific. A great opportunity for attaining cultural understanding is remembering that even specialised healthcare providers can expand their awareness of other cultures and learn from those they serve. Biomedical values are not always reconcilable with other cultural values. Sensitivity should be given to individual's previous experience with the medical system and models for achieving health.

(C) Recognise the value placed on trust and respect.

Sincerity is recognised as an emotional strength. Focused communication, including listening and noting responses, strengthens a relationship built on trust and respect. If trust is given to caregivers by a Mi'kmaw as a way of respecting their role, the trust should be protected, never abused or disregarded.

(D) Be aware of non-verbal communication and cues Minimal eye contact may not indicate discomfort. Care providers can share information to for discussion.

(E) Consider the role of religious beliefs and spirituality. encourage discussion rather than directly asking personal, intrusive questions.

Spirituality, in particular feelings toward life, death and afterlife, hold varying degrees of importance. When traditional views of going to the spirit world are identified, providers should recognise that death is viewed as a transition from one state to another and not with the same finality as other worldviews concerning death. Spiritual and religious beliefs play an important role in framing processes and choices relating to values enacted at this time. 
choices and preferences. The integration of spirit as a part of healing occurs through the inseparable interrelationships among self, family and community. ${ }^{19}$

The Mi'kmaq community approaches life in the context of a worldview which may include traditional medicine and an interconnectedness of physical, mental, spiritual and environmental well-being. Ruth Whitehead described the family in pre-European contact times as 'the centre of the universe' and emphasised the role of alliances created by birth, marriage or adoption as being priorities in life. The collective mindset that frames kinship is still a cultural imperative for the Mi'kmaq and characterises the role of family and community in a Mi'kmaq worldview. Consequently, family and community play a predominant role in ethical decision-making and confidentiality. The preferred process for leaving the physical world is in the company of loved ones and choices are made with the family either involved or kept in mind.

Community preparation and participation in the journey to the spirit world is important. Wakes and funerals are commonly accepted as social gatherings where people converse openly, laugh, share stories and celebrate life which supports the tradition of strong family and community connectedness. Healing or prayer circles as well as ceremonial sweats may be held when it is anticipated that the person's journey to the spirit world is eminent. According to Angela Robinson, ${ }^{19}$ the tenet, 'No one is born alone and no one should die alone,' is central to Mi'kmaq understanding of not going through this journey alone. There is a community of faith that binds individuals to one another as well as to a greater collective.

The 'spirit road' is sometimes referred to by the Mi'kmaq as a journey that every person must take. It is not travelled alone, but rather in the company of the ancestors, linking the individual's journey with those who came before. Drumming; praying to the four directions; lighting sage, tobacco or sweet grass; and singing or chanting sometimes are included by individuals accompanying the person in this journey. Scared fires and pipe ceremonies are also traditions that communities may partake in collectively during this transition. End of life is not so much its own period but a continuation of the realities that there are many levels of existence. Combined with the importance of family and community, the perception of spirit is linked to a strong sense of faith in both the life and death process. 'Letting the spirit go' refers to family, friends, and loved ones encouraging or giving the sick person opportunity to let their life spirit go to the 'other world.'

As a result of this perspective, healthcare providers should not restrict the number of visitors, as family means more than immediate family members. Prohibiting visitors from bringing sacred objects or prohibiting ceremonies that enable an individual to travel to the spirit world is also problematic.
Respect for handling sacred objects or personal belongings and knowing who can handle certain objects is important. A woman who is on her moon time (menses) should not touch sacred objects because women are believed to be very powerful during this time. Culturally competent advocacy on the part of healthcare providers is fundamental to providing care that is grounded in the understanding of this journey to the spirit world.

Healthcare providers can play a critical role in providing an environment that positively affects the individual and those in attendance at end of life by learning the values and beliefs of the individuals and families who are willing to accept their care.

Historically, coping with illness, bereavement and pain is considered acceptable when met with eventemperedness and suppression of grief rather than public displays of despair and inconsolable grief. ${ }^{15}$ Whitehead spoke of the value of patience which was often associated with the importance placed on not complaining about ailments. This was perceived as a survival mechanism or a way of being stoic in the face of disaster or illness. However, Mi'kmaq people do not view the English term stoic as being an adequate description of their cultural teaching and socialisation. Historically, Mi'kmaq suffered without showing the effect of misfortune and to do the contrary was believed to be the height of immaturity or a means of drawing attention, even causing harm. Complaining was perceived as being selfish and was considered to have the effect of making others depressed.

Special respect and deference for elders is a fundamental given in Mi'kmaq communities. Some respected elders are known for their knowledge and wisdom of oral traditions and this may not necessarily be related to age. Elders are well known and play a significant role in the community. Healthcare providers must build relations with respected elders in the community as the relationship is seen as a partnership for addressing culturally competent care for community members.

Historically, care giving was defined within the context of reciprocity, and was often experienced throughout the lifespan. Today, there are differences in the availability of supports that exist on and off reserve which has led to changes in care, particularly around the role of the community in providing assistance and the role of family in providing respite care. $^{9}$

A sense of family, community and traditional views of spirituality frame the view of health among Mi'kmaq. There is a rooted belief in the strength of prayer and faith in its ability to cure as much as any intervention. More value may be placed on quality of life rather than on pursuit of a cure when life balance and wellness are a part of the holism that is ethically equated with mental, physical, emotional and spiritual health. ${ }^{20}$ 
Aboriginal worldviews see health as more than a lack of disease. Health is linked to the economy, the land, community and culture. ${ }^{21}$ Emphasis on biomedical treatment through technological interventions may be associated with a colonial approach to health, while at the same time diverse perspectives regarding aggressive treatment and technology abound. ${ }^{22} \mathrm{~A}$ study reported that $66 \%$ of a sample of 100 Mi'kmaw patients at a community health centre used Mi'kmaq medicine. ${ }^{23}$ Of those, $24.3 \%$ reported using it as a first-line of treatment, $92.4 \%$ had not discussed this with their physician and $31.8 \%$ said they believed Mi'kmaq medicine was better overall than Western medicine. Non-Mi'kmaq healthcare providers should not assume that someone in their care is following treatment measures prescribed by Western medicine and should openly discuss this with clients in a nonjudgemental manner. It is important for non-Mi'kmaq healthcare providers to understand and appreciate the worldview, spirituality, role of family and community, and communication norms of Mi'kmaw individuals with whom they are working, and to strive for an alignment of values in the provision of care.

\section{Language}

The words that are used and a lack of common understanding of terms can lead to miscommunication and misinterpretation of needs and services. For example, terms such as home care, supportive care and palliative care are sometimes used interchangeably by federal and provincial service providers even though they may imply different ideas.

Language barriers also affect the understanding of care plans. Even if interpretation services are available, they may not be accessed because persons are not identified as having this need since the Mi'kmaq generally have some level of understanding of English, some Mi'kmaq may not speak Mi'kmaq or because interpreters may not be readily available. Furthermore, interpretation services may not adequately handle the nuances of Mi'kmaq terms and the wording used by non-Mi'kmaw healthcare providers.

It is important to note that traditional Mi'kmaq do not use the term 'end of life' since they view the present life as a continuum into a next life. Mi'kmaq terms, which are not well understood by non-Mi'kmaq care providers, are very relevant. Three of these (apiksiktatulti, nemu'ltus and salite) are introduced in table 1. Mi'kmaq traditions associated with the journey to the spirit world are culture-specific and individually determined.

Beyond access to publicly-funded health services and historic Mi'kmaq ways of caring for each other, there can be an additional dimension. Jesuit priests introduced the Mi'kmaq to Roman Catholicism which remains important to varying degrees among the Mi'kmaq in terms of both values and language; for example, heaven may be the term used for the spirit world by some Mi'kmaq.

\section{DISCUSSION}

By separating and reflecting on the issues of jurisdiction and cultural understanding, progress can be made toward greater cultural competency. Inclusion of indigenous ways of knowing about health is critically important for ensuring cultural competency. Non-Aboriginal healthcare providers often discuss and provide supportive and palliative care from a Eurocentric approach $^{24}$ either due to lack of knowledge or lack of system support to provide culturally competent supportive and palliative care. Cultural competency transcends cultural understanding. It includes ensuring that the healthcare system is inclusive of different worldviews in the planning, implementation and evaluation of healthcare services.

The authors recommend that indigenous leaders undertake a more indepth study of supportive and palliative care needs in their communities by using an integrative framework to guide this research. ${ }^{25}$ This paper demonstrates the need for understanding across conceptualisations and is in accord with the report of the Canadian Hospice Palliative Care Association, which advises building on strengths and working together to improve Aboriginal hospice palliative care. ${ }^{26}$

The authors recommend that consideration be given to having palliative care researchers work with the Mi'kmaq using research processes that respect the community. The purpose would be to explore how current perspectives on palliative and supportive care might be enhanced or reconstructed through a greater understanding of the Mi'kmaq ways.

\section{CONCLUSION}

The identification of both jurisdictional issues and cultural understanding in the historic context of the Mi'kmaq is important for enhancing the cultural competency of non-Mi'kmaq care providers. Best practice indigenous supportive and palliative care may be contrary to conventional Western healthcare assumptions and practice. Through reflection on the situation of Nova Scotia's Mi'kmaq, non-indigenous healthcare providers can assess how they might critique and increase their cultural competency. This paper will hopefully raise awareness of the need to more broadly understand and incorporate an indigenous cultural context when providing supportive and palliative care.

Acknowledgements The authors thank and acknowledge the following for sharing their insight, understanding and guidance in the preparation of this manuscript: Theresa Meuse, Mi'kmaq educator, advisor and author; Sister Veronica Matthews, retired Aboriginal nurse and Mi'kmaq Elder; Charlotte (Loppie) Reading, Human and Social Development, University of Victoria, and formerly School of Health and 
Human Performance, Dalhousie University; Peter L Twohig, Canada Research Chair in Atlantic Canada Studies, Saint Mary's University; Angela Robinson, Social/Cultural Studies, Anthropology, Sir Wilfred Grenfell College, Memorial University; and Ruth Whitehead, Curator Emerita, Nova Scotia Museum.

Contributors GJ was responsible for overall conceptualisation of the paper and guidance especially in relation to palliative and end of life care for vulnerable populations as well as critical reflection, interpretation and editing. AV advised on Mi'kmaq information sources, conceptualisation and design, and assisted with the critical review, understanding of the Mi'kmaq culture, liaison with Mi'kmaq consultants and editing of the manuscript. SP carried out the initial literature review and meetings with strategic informants, prepared the first draft of this paper and contributed to revisions. All authors contributed to the literature review and have read and approved this final manuscript. GJ and AV jointly are the guarantors for the content of this paper based on their respective areas of expertise and through ongoing consultation with two Mi'kmaq consultants.

Funding This report was carried out with support from a Canadian Institutes for Health Research Interdisciplinary Capacity Enhancement for vulnerable populations grant number FRN-80067.

\section{Competing interests None.}

Provenance and peer review Not commissioned; externally peer reviewed.

Open Access This is an Open Access article distributed in accordance with the Creative Commons Attribution Non Commercial (CC BY-NC 3.0) license, which permits others to distribute, remix, adapt, build upon this work non-commercially, and license their derivative works on different terms, provided the original work is properly cited and the use is noncommercial. See: http://creativecommons.org/licenses/ by-nc/3.0/

\section{REFERENCES}

1 Pottinger A, Pervolaris A, Howes D. The end of life. In: Srivastva R, eds. The Health Care Professional's Guide to Clinical Cultural Competence. Toronto: Elsevier 2007:227-237.

2 Kagawa-Singer M, Blackhall LJ. Negotiating cross-cultural issues at the end of life: 'You got to go where he lives'. JAMA 2001;286:2993-3001.

3 Fox RC. Cultural competence and the culture of medicine. N Engl J Med 2005;353:1316-1319.

4 Maddalena V. Cultural competence and holistic practice: implications for nursing education, practice, and research. Holist Nurs Pract 2009;23:153-157.

5 Statistics Canada. Aboriginal identity population by age groups, median age and sex, 2006 counts for both sexes, for Canada, provinces and territories $-20 \%$ sample data. Aboriginal Peoples - Data Table 2010. http://www12.statcan.ca/ census-recensement/2006/dp-pd/hlt/97-558/pages/page.cfm? Lang $=\mathrm{E} \& \mathrm{Geo}=\mathrm{PR} \&$ Code $=01 \&$ Table $=1 \&$ Data $=$ Count $\&$ Sex $=1 \&$ Age $=1 \&$ StartRec $=1 \&$ Sort $=2 \&$ Display $=$ Page (accessed 9 August 2011).

6 Battiste M. Unfolding the lessons of colonization. In: Battiste M, eds. Reclaiming Indigenous Voice and Vision. Vancouver: UBC Press 2000:xvi-xxx.

7 Kelley ML. An indigenous issue: why now? J Palliat Care 2010;26:5.

8 Hanson G. Working with elders and advisers in Aboriginal palliative care: a story from the Canadian Pallium Project. J Palliat Care 2010;26:37-40.

9 Twohig PL. Colonial care: medical attendance among the Mikmaq in Nova Scotia. Can Bull Med Hist 1996; 13:333-353.

10 Whelan TJ, Mohide EA, Willan AR, et al. The supportive care needs of newly diagnosed cancer patients attending a regional cancer center. Cancer 1997;80:1518-1524.

11 Supportive Care Guidelines Development Group. Supportive care evidence-based series and practice guidelines. Cancer Care Ontario, 2011. http://www.cancercare.on.ca/cms/One.aspx? portalId=1377\&pageId=10397 (accessed 18 August 2011).

12 National Institute of Health (NIH). NIH State-of-the-Science Conference Statement on Improving End-of-Life Care. NIH Consensus and State-of-the-Science Statements, 2004 http:// consensus.nih.gov/2004/2004EndOfLifeCareSOS024PDF.pdf (accessed 9 August 2011).

13 Gahagan J, Loppie C, MacLellan M, et al. Caregiver Resilience and the Quest for Balance: A Report on Findings from Focus Groups. Healthy Balance Research Program 2004. http://www.acewh.dal.ca/ eng/reports/TeamQ-web.pdf (accessed 9 August 2011).

14 Williams AM, Crooks VA, Whitfield K, et al. Tracking the evolution of hospice palliative care in Canada: a comparative case study analysis of seven provinces. BMC Health Serv Res 2010;10:147.

15 Loppie C, Wien F. Our Journey: First Nations Experience in Navigating Cancer Care. Final Report on behalf of the Mi'kmaq Health Research Group. Halifax: Cancer Care Nova Scotia, 2005.

16 Nova Scotia Department of Health. A Cultural Competence Guide for Primary Health Care Professionals in Nova Scotia. Primary Health Care Section, Nova Scotia: Department of Health, 2005. http://www.healthteamnovascotia.ca/ cultural_competence/Cultural_Competence_guide_for_ Primary_Health_Care_Professionals.pdf (accessed 9 August 2011).

17 Durst D, Bluechardt M. Aboriginal People with Disabilities: A Vacuum in Public Policy. The Saskatchewan Institute of Public Policy, Briefing Notes, 2004 http://www.uregina.ca/sipp/ documents/pdf/BN6_Aboriginal\%20Persons\%20with\% 20Disabilities.pdf (accessed 23 March 2006).

18 Lavoie JG, Forget E, O’Neil JD. Why equity in financing first nations on-reserve health services matters: findings from the 2005 national evaluation of the health transfer policy. Healthc Policy 2007;2:79-96.

19 Robinson A. Ta'n Teliktlamsitasit (Ways of Believing). Toronto: Pearson Education Canada 2005.

20 Russell SS. Cultural consideration in the end-of-life. Med-Surg Matters 2005;14:6-8.

21 Ellerby JH, McKenzie J, McKay S, et al. Bioethics for clinicians: 18. Aboriginal cultures. CMAJ 2000;163:845-850.

22 Dokis L. Staying true to traditions can improve health. Windspeaker 2004;21:16. 
23 Cook SJ. Use of traditional Mi'kmaq medicine among patients at a First Nations community health centre. Can J Rural Med 2005;10:95-99.

24 Baydala A, Hampton M, Kinunwa L, et al. Death, dying, grieving, and end of life care: understanding personal meanings of Aboriginal friends. The Humanistic Psychologist 2006;34:159-176.

25 Prince H, Kelley ML. An integrative framework for conducting palliative care research with First Nations communities. J Palliat Care 2010;26:47-53.
26 Canadian Hospice Palliative Care Association. Moving Forward by Building on Strengths: A Discussion Document on Aboriginal Hospice Palliative Care in Canada. First Nations and Inuit Health, First Nations and Inuit Home and Community Care Program, Health Canada, 2007. http://www. cspcp.ca/english/Moving\%20Forward\%20by\%20Building $\%$ 20on\%20Strengths\%20A\%20Discussion\%20Document $\% 20$ on \%20Aboriginal\%20Hospice\%20Palliative\%20Care\%20in\% 20Canada.pdf. (accessed 25 August 2011) 